\title{
Union Health and Family Welfare Centers in Chittagong and Munshiganj: Are they ready to provide 24-hour normal delivery services?
}

Md. Noorunnabi Talukder

Population Council

Ubaidur Rob

Population Council

A.K.M. Zafar Ullah Khan

Population Council

Forhana Rahman Noor

Population Council

Shongkour Roy

Population Council

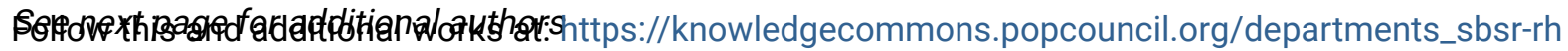

Part of the Demography, Population, and Ecology Commons, Family, Life Course, and Society Commons, International Public Health Commons, Maternal and Child Health Commons, and the Women's Health Commons

How does access to this work benefit you? Let us know!

\section{Recommended Citation}

Talukder, Md. Noorunnabi, Ubaidur Rob, A.K.M. Zafar Ullah Khan, Forhana Rahman Noor, Shongkour Roy, and Afsana Fatema Noor. 2015. "Union Health and Family Welfare Centers in Chittagong and Munshiganj: Are they ready to provide 24-hour normal delivery services?" Dhaka: Population Council. 


\section{Authors}

Md. Noorunnabi Talukder, Ubaidur Rob, A.K.M. Zafar Ullah Khan, Forhana Rahman Noor, Shongkour Roy, and Afsana Fatema Noor 


\section{Union Health and Family Welfare Centers in Chittagong and Munshiganj Are They Ready to Provide 24-Hour Normal Delivery Services?}

MD. NOORUNNABI TALUKDER

UBAIDUR ROB

A.K.M. ZAFAR ULLAH KHAN FORHANA RAHMAN NOOR

SHONGKOUR ROY

AFSANA FATEMA NOOR
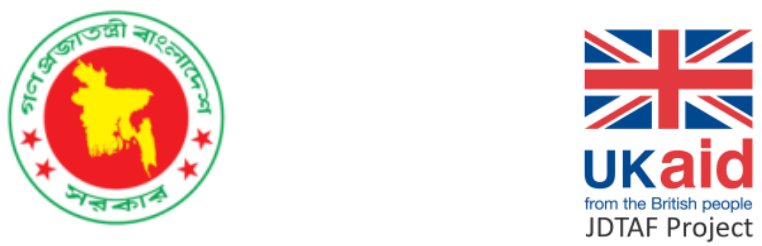

POPULATION COUNCIL 


\section{Dopountrow \\ Ideas. Evidence. Impact.}

The Population Council confronts critical health and development issues-from stopping the spread of HIV to improving reproductive health and ensuring that young people lead full and productive lives. Through biomedical, social science, and public health research in 50 countries, we work with our partners to deliver solutions that lead to more effective policies, programs, and technologies that improve lives around the world. Established in 1952 and headquartered in New York, the Council is a nongovernmental, nonprofit organization governed by an international board of trustees.

\section{Population Council}

Bangladesh Country Office

House 15B, Road 13, Gulshan 1

Dhaka 1212, Bangladesh

Email: info.bangladesh@popcouncil.org

popcouncil.org

Suggested citation: Talukder, M.N., U. Rob, A.K.M.Z.U. Khan, F.R. Noor, S. Roy, and A.F. Noor. 2015. "Union Health and Family Welfare Centers in Chittagong and Munshiganj: Are They Ready to Provide 24-Hour Normal Delivery Services?" Dhaka: Population Council. 


\section{TABLE OF CONTENTS}

List of Tables and Figures iv

List of Abbreviations $\quad \mathrm{V}$

Acknowledgements

Summary vii

\begin{tabular}{l|l} 
I INTRODUCTION & 1
\end{tabular}

II METHODOLOGY 1

III FINDINGS FROM THE FACILITY ASSESSMENT 2

A. Physical Infrastructure $\quad 2$

- Amenities 4

- $\quad$ Labor room 6

B. Human Resources $\quad 7$

C. Equipment, Logistics, and Supplies 10

D. Service Delivery 11

- Normal delivery services 11

- Referral 12

- Infection prevention 12

$\begin{array}{ll}\text { E. Management } & 13\end{array}$

IV FINDINGS FROM THE PROVIDER SURVEY 15

A. Age, recruitment, and placement 15

$\begin{array}{ll}\text { B. Provider competence } & 16\end{array}$

C. Problems faced by providers in performing job 23

$\begin{array}{lll}V & \text { DISCUSSIONS AND RECOMMENDATIONS }\end{array}$ 


\section{LIST OF TABLES AND FIGURES}

Table 1: Types of Union Health and Family Welfare Centers (percent)

Table 2: Distribution of usable rooms in Union Health and Family Welfare Centers (percent)

Table 3: Utilities and amenities in Union Health and Family Welfare Centers (percent)

Table 4: Condition of toilets in Union Health and Family Welfare Centers (percent)

Table 5: Physical condition of waiting and FWV rooms in Union Health and Family Welfare Centers (percent)

Table 6: Situation of the labor room in Union Health and Family Welfare Centers (percent)

Table 7: Training received by Family Welfare Visitors (percent)

Table 8: Management of Union Health and Family Welfare Centers (percent)

Table 9: Distribution of FWVs by age groups (number)

Table 10: Working experience of FWVs (number)

Table 11: Satellite clinic services provided by FWVs (number)

Table 12: Counseling skills of FWVs on maternal health services (number)

Table 13: Examinations performed by FWVs to ensure pregnancy (number)

Table 14: Knowledge of FWVs on the types of examinations a woman needs during first antenatal care visit (number)

Table 15: Knowledge of FWVs on possible complications during pregnancy, during delivery, and after delivery (number)

Table 16: Knowledge of FWVs on five danger signs of pregnancy (number)

Table 17: Knowledge of FWVs on signs and symptoms of newborn complications (number)

Table 18: Awareness of FWVs on basic preventive care for newborn (number)

Table 19: Clinical skills of FWVs on maternal health care (number)

Table 20: Pregnancy complications reported by FWVs for referral (number)

Table 21: Facilities to which clients are referred (number)

Table 22: Problems experienced by FWVs while working in the facility (number)

Figure 1: Physical infrastructure of Union Health and Family Welfare Centers by number of rooms (percent)

Figure 2: Labor and recovery rooms in Union Health and Family Welfare Centers (percent)

Figure 3: Staff composition at the Union Health and Family Welfare Center (percent)

Figure 4: Accommodation and residential status of service providers (percent)

Figure 5: Supply of DDS kits in the previous month (percent)

Figure 6: Availability of poster or wall writing for mass viewing (percent)

Figure 7: Reasons for not providing normal delivery services (percent)

Figure 8: Clients referred to higher-level facilities from the UHFWC (percent)

Figure 9: Practice of sterilization of equipment and waste management (percent)

Figure 10: Status of Union Health and Family Planning Committees (percent) 


\section{LIST OF ABBREVIATIONS}

$\begin{array}{ll}\text { BCC } & \text { Behaviour Change Communication } \\ \text { DDS } & \text { Drug and Dietary Supplement } \\ \text { DGFP } & \text { Directorate General of Family Planning } \\ \text { DH } & \text { District Hospital } \\ \text { EmONC } & \text { Emergency Obstetric and Newborn Care } \\ \text { ENC } & \text { Essential Newborn Care } \\ \text { FPI } & \text { Family Planning Inspector } \\ \text { FWV } & \text { Family Welfare Visitor } \\ \text { IUD } & \text { Intra-uterine Device } \\ \text { MCWC } & \text { Mother and Child Welfare Center } \\ \text { MR } & \text { Menstrual Regulation } \\ \text { SACMO } & \text { Sub-Assistant Community Medical Officer } \\ \text { UHC } & \text { Upazila Health Complex } \\ \text { UHFPC } & \text { Union Health and Family Planning Committee } \\ \text { UHFWC } & \text { Union Health and Family Welfare Center }\end{array}$




\section{ACKNOWLEDGEMENTS}

Population Council would like to express sincere gratitude to the UK Department for International Development (UKaid) for their financial assistance for carrying out the project "Strengthening Union Health and Family Welfare Centers for Providing Roundthe-Clock Normal Delivery Services" under which this situation analysis was conducted.

Special thanks are due to the district-level and upazila-level government program managers, including Deputy Directors of Family Planning, Upazila Health and Family Planning Officers, Upazila Family Planning Officers, and Medical Officers-Maternal and Child Health and Family Planning for their cooperation to conduct the assessment. We are also grateful to service providers of UHFWCs who participated in the assessment. In addition, we would like to convey our sincere thanks to the study team who carried out the situation analysis activity.

We are grateful to Dr. Mohammed Sharif, Director, Maternal and Child Health Services and Dr. Tapash Ranjan Das, Deputy Director, Maternal and Child Health Services from the Directorate General of Family Planning for their guidance and cooperation at every step of the study.

We gratefully acknowledge the cooperation of Mr. Devashish Banerji, Program ManagerService Provider for Joint Donor Technical Assistance Fund, Crown Agents Bangladesh in facilitating funding for the project.

Finally, we are thankful to Mr. Dipak Shil, Director of Administration, Finance and Human Resources at the Population Council Bangladesh Office for the financial management of this project. 


\section{SUMMARY}

To date, the Directorate General of Family Planning (DGFP) of Ministry of Health and Family Welfare has established approximately 3,900 Union Health and Family Welfare Centers (UHFWCs) in rural areas providing: family planning; menstrual regulation; vaccinations; and general, reproductive, and maternal health services six days a week. About 1,500 UHFWCs have been upgraded with the necessary staff and equipment to provide normal delivery services round-the-clock in rural areas. Yet, Family Welfare Visitors (FWVs) posted at UHFWCs perform only 0.3 percent of deliveries. In rural areas, 69 percent of deliveries occur at home assisted largely by unskilled or traditional birth attendants (64\%). This means that UHFWCs and FWVs are not optimally utilized to increase the rate of institutional deliveries.

It is important to understand the processes needed to provide 24-hour normal delivery services at UHFWCs. To address this issue, the Population Council is providing technical assistance to the DGFP to implement an Operations Research project that tests the effectiveness of a model to provide round-the-clock normal delivery services in 24 UHFWCs in Chittagong and Munshiganj districts. UKaid, through the Crown Agents, provided funding for this project. As a part of the project, a situation analysis comprising a health facility assessment and provider survey was conducted. To explore the status of the UHFWCs in two intervention districts, a total of 174 facilities were assessed (Chittagong: 123, Munshiganj: 51). In addition, a survey was conducted with 27 FWVs (Chittagong: 15, Munshiganj: 12) who provide normal delivery services at 24 intervention UHFWCs to understand their technical competence in terms of knowledge and capacities in providing round-the-clock normal delivery services. This report describes the outcome of this situation analysis activity.

\section{Findings}

Several gaps in the existing capacity of UHFWCs for providing round-the-clock delivery services were identified through situation analysis. Necessary inputs are broadly described in five categories: physical infrastructure, human resources, equipment and supplies, management, and referral.

\section{PHYSICAL INFRASTRUCTURE}

Three types of UHFWCs operated by the DGFP were found in terms of physical structure. More than 60 percent of UHFWCs were one storied, 32 percent were two storied and only 5 percent were three storied. Largely, UHFWCs had separate rooms for the staff along with delivery and recovery rooms. Rooms are not fully furnished, nevertheless service provision is possible. 
In some cases, infrastructure was in poor condition. There was lack of essential utilities, e.g., supply of water and electricity. Toilet facilities were somewhat satisfactory. On an average, there were three toilets in each facility and one-fifth had separate toilets for males and females.

\section{HUMAN RESOURCES}

- Existing human resources are not adequate for performing delivery services at the UHFWC, nor for providing round-the-clock services. FWVs did not have adequate training to provide normal delivery services. Only half of the FWVs had training on midwifery and a few FWVs were trained on active management of the third stage of labor. Not all FWVs had adequate knowledge on obstetric danger signs. Largely, FWVs were not skilled to use partograph.

- Identification of danger signs of pregnancy and conditions for high-risk pregnancy is subject to the correct knowledge and skills of providers. The composite skills score reveals inadequate knowledge of FWVs on pregnancy and delivery complications. The assessment also reveals an inadequacy in the skills of FWVs in the management of essential obstetric complications. Gaps in knowledge and skills on obstetric complications can be addressed through refresher training and technical monitoring.

- In essential counseling skills, e.g., general health counseling, birth planning counseling and advice during discharge after delivery, FWVs failed to score a high competency level. Providers need counseling training as part of the program and professional monitoring from higher level.

- There is a serious deficiency in knowledge and skills of basic neonatal care of FWVs (a maximum composite score of 0.50 out of 1.00). Training on "essential newborn care" and professional monitoring from higher level are the possible avenues to address inadequacy in FWVs' knowledge and skills on newborn care.

- FWVs are largely non-residential despite having options for accommodation in the facility building. It is necessary to ensure that FWVs who are the first contact for pregnant women stay at residence attached to the UHFWC for providing 24-hour normal delivery services.

\section{EQUIPMENT AND SUPPLIES}

- Equipment and logistics in the labor and recovery rooms were not fully available in the facilities which need attention as these two rooms are critical for providing normal delivery services. 
- In a few UHFWCs, there is a rest room or post-operative room for intra-uterine device, menstrual regulation and delivery clients. None of the UHFWCs reported having a furnished recovery room. A recovery room with at least two beds is necessary to provide round-the-clock delivery services.

- Another major problem for the labor room is that a few UHFWCs had a generator as an alternate source of electricity. For 24-hour services, it is necessary to ensure uninterrupted electricity.

- Educational materials on maternal health, such as flipchart, brochure, poster and wall writing, were inadequate. The materials are important tool for raising awareness among service recipients.

\section{MANAGEMENT}

- Encouragingly, monthly reporting from UHFWCs to higher levels was found regular in all UHFWCs.

- About 80 percent of the unions have Union Health and Family Planning Committee (UHFPC), but only 10-18 percent of those committees were found functional where monthly meetings were held, mostly irregularly. None of the committees was empowered to oversee the activities of the UHFWC and to contribute to the UHFWC maintenance. Initiatives should be taken to reactivate the existing committees or expedite the process of forming committees for the UHFWCs having no such committee with appropriate supervisory and financial authority.

- UHFWCs should publicize their 24-hour normal delivery services, including the names of service providers, to capture the local population's attention.

\section{REFERRAL}

- There should be specific direction on where to refer pregnant woman if complications are identified at the UHFWC. UHFWCs should have a functional linkage with both basic emergency obstetric and newborn care (EmONC) and comprehensive EmONC facilities for referring complicated cases where appropriate. All Upazila Health Complexes $^{1}$, which are the first referral center for FWVs posted at UHFWCs, should be strengthened with appropriate human resources and equipment to conduct cesarean deliveries and provide emergency obstetric care.

\footnotetext{
${ }^{1}$ Currently, one-third of the Upazila Health Complexes conduct cesarean deliveries and provide comprehensive EmONC.
} 


\section{Way Forward}

Limited availability of the FWV at the UHFWC (4 out of 6 working days), who is the only provider for conducting delivery services, is the key programmatic challenge to provide 24hour normal delivery services from the UHFWC. Moreover, FWVs are not skilled to provide basic EmONC. The UHFWC requires a provider who should be adequately trained in midwifery care to address essential functions of EmONC and to make referral to the Upazila Health Complex and higher-level facilities for complications management and cesarean deliveries. A new cadre of "midwife" can be created to address the maternal health care needs of the growing female population in rural areas.

In rural Bangladesh, more than half of deliveries take place at home while union-level facilities remain underutilized. It has been estimated that approximately 400 child births take place in a union per year of which 15 percent require cesarean sections from higher-level facilities. As per global standard protocol, a trained mid-level provider (e.g., FWV at the UHFWC) can perform 175 deliveries annually, which comprises half of the normal deliveries of a union. There is no alternate to increase facility-based delivery in rural areas to reduce maternal health risks; therefore, it is necessary to strengthen UHFWCs with skilled human resources, service provision, logistics and supplies, and local level management. 


\section{INTRODUCTION}

The government of Bangladesh has established around 3,900 Union Health and Family Welfare Centers (UHFWCs), which are in proximity to the rural populations, providing: family planning; menstrual regulation; vaccinations; and general, reproductive, and maternal health services. In this facility, primarily two paramedics, Sub-Assistant Community Medical Officer (SACMO) and Family Welfare Visitor (FWV), provide outdoor services six days a week. About 1,500 UHFWCs have been upgraded with necessary human resources and equipment to provide normal delivery services round-the-clock. Yet, FWVs posted at the UHFWC perform only 0.3 percent of deliveries. In Bangladesh, about 63 percent of deliveries occur at home assisted largely by unskilled or traditional birth attendants ${ }^{2}$. This means that UHFWCs and FWVs are not optimally utilized to increase the rate of institutional deliveries.

In this context, the Population Council is providing technical assistance to the Directorate General of Family Planning (DGFP) to implement an Operations Research project which tests effectiveness of a model to provide round-the-clock normal delivery services in 24 UHFWCs in Chittagong and Munshiganj districts in Bangladesh. UKaid, through the Crown Agents, provided funding for this project. As a part of the project, a situation analysis comprising a health facility assessment and provider survey was conducted. To explore the status of facilities, all UHFWCs operated by the DGFP from two intervention districts were assessed. In addition, a survey was conducted with FWVs who provide normal delivery services at 24 intervention UHFWCs to assess their maternal health knowledge and capacities in providing round-the-clock normal delivery services. This report is the outcome of this situation analysis activity.

\section{METHODOLOGY}

Chittagong is one of the largest districts in Bangladesh consisting of 14 upazilas and 198 unions. On the other hand, Munshiganj district consists of 6 upazilas and 68 unions. At the union level, there is a government health facility operated by either "Family Planning" department or "Health" department. However, not all unions have a health facility. Out of 266 unions, 174 unions have UHFWCs operated by the DGFP and 31 unions have facilities operated by the Directorate General of Health Services. As Chittagong district is geographically larger than Munshiganj district, larger number of UHFWCs were assessed in Chittagong district (123) compared to Munshiganj district (51).

A checklist was developed and pre-tested for assessing the status of UHFWCs. Skilled persons were recruited and trained on the checklist for data collection. Data collectors conducted the assessment during December 2014 to January 2015 by visiting UHFWCs and interviewing concerned service providers.

\footnotetext{
2 National Institute of Population Research and Training (NIPORT), Mitra and Associates, ICF International. 2015. Bangladesh Demographic and Health Survey 2014: Key Indicators. Dhaka, Bangladesh and Rockville, MD: NIPORT, Mitra and Associates, and ICF International.
} 
In addition, a provider survey was conducted to assess the technical competence in terms of knowledge and skills of FWVs who provide normal delivery services and to explore their experience on infrastructural challenges in providing round-the-clock normal delivery services from UHFWCs. A total of 27 FWVs from the intervention UHFWCs of two districts (Chittagong: 15, Munshiganj: 12) were interviewed in March 2015. Experienced and trained data collectors interviewed FWVs at their working place.

\section{FINDINGS FROM THE FACILITY ASSESSMENT}

This section provides a summary of the assessment of UHFWCs in two districts, focusing on important features of physical infrastructure, human resources, equipment and supplies, service delivery, and management. The findings describe what is actually happening at UHFWCs in terms of inputs and processes in two districts in Bangladesh.

\section{A. Physical Infrastructure}

Information was collected on the building, utilities and communication. It was found that more than 60 percent of the assessed UHFWCs are one storied and one-third are two storied. Overall, five percent of UHFWCs were three storied with Munshiganj district having more three-storied facilities than Chittagong (Table 1). The decade of 1980s has witnessed the construction of the majority of UHFWCs (55\%) and another 22 percent were established before 1980 while the remaining UHFWCs were built after 1990 (not shown).

Table 1: Types of Union Health and Family Welfare Centers (percent)

\begin{tabular}{lrrr}
\hline Type & Chittagong & Munshiganj & Total \\
One storied & 64.2 & 62.8 & 63.8 \\
Two storied & 32.6 & 29.4 & 31.6 \\
Three storied & 3.2 & 7.8 & 4.6 \\
$\mathrm{~N}$ & $\mathbf{1 2 3}$ & $\mathbf{5 1}$ & $\mathbf{1 7 4}$ \\
\hline
\end{tabular}

UHFWCs had a minimum of five rooms: waiting space and rooms for FWV, SACMO, Family Planning Inspector (FPI), and a room for inserting the intra-uterine device (IUD), performing menstrual regulation (MR) and conducting normal deliveries. In addition, some UHFWCs had a pharmacy, a recovery room, a storeroom and/or doctor's room. 
Figure 1: Physical infrastructure of Union Health and Family Welfare Centers by number of rooms (percent)

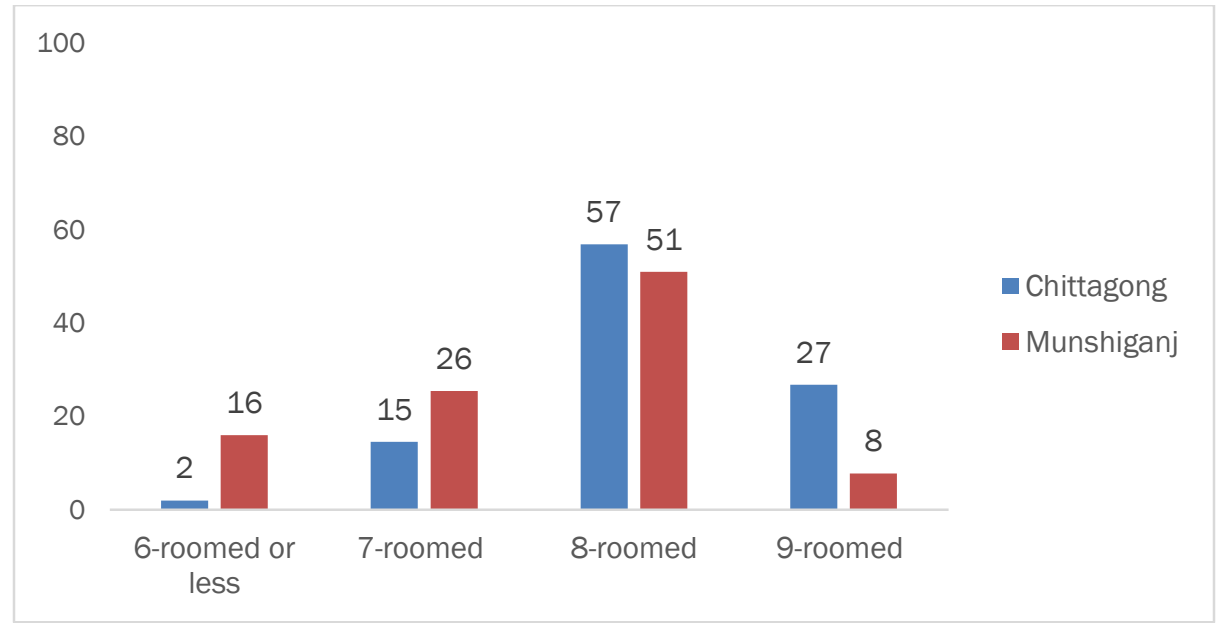

There was variation in the number of rooms across facilities. Half of the UHFWCs were found to have eight rooms. There were more nine-roomed UHFWCs in Chittgaong than in Munshiganj (27\%, $8 \%$ respectively) while Munshiganj had more facilities with seven rooms compared with Chittagong. Facilities with six rooms or less were nearly absent in Chittagong, yet one in every six facilities in Munshiganj had six rooms (Figure 1).

Table 2 shows the distribution of usable rooms in UHFWCs. Nearly all facilities had a waiting space, and separate rooms for FWV, SACMO and FPI. Nearly 90 percent of the UHFWCs had usable labor/delivery room along with nearly two-thirds or more having recovery room. Half of the facilities had a pharmacy and a store. Variations in the distribution of rooms between districts are small.

Table 2: Distribution of usable rooms in Union Health and Family Welfare Centers (percent)

\begin{tabular}{lrrr} 
Room & Chittagong & Munshiganj & Total \\
Waiting space & 97.6 & 94.1 & 96.6 \\
SACMO & 96.7 & 100.0 & 97.7 \\
FWV & 94.3 & 94.1 & 94.3 \\
FPI & 93.5 & 96.1 & 94.3 \\
Pharmacy & 52.8 & 56.9 & 54.0 \\
Store & 65.9 & 51.0 & 61.5 \\
Labor/Delivery & 88.6 & 86.3 & 87.9 \\
Recovery & 76.4 & 62.7 & 72.4 \\
N & 123 & 51 & 174 \\
\hline
\end{tabular}

$\mathrm{SACMO}=$ Sub-Assistant Community Medical Officer. FWV=Family Welfare Visitor. FPI= Family Planning Inspector. 


\section{AMENITIES}

Table 3 shows the availability of utilities and amenities in UHFWCs. A significant number of UHFWCs had neither electricity nor water supply. More than 70 percent of the facilities had electricity with no large differences between the districts. Overall, one-third of the facilities reported the unavailability of water at the facility compound. More facilities in Chittagong than Munshiganj were required to bring water away from the facility (38\% and $24 \%$ respectively).

Table 3: Utilities and amenities in Union Health and Family Welfare Centers (percent)

\begin{tabular}{lrrr}
\hline Utilities and amenities & Chittagong & Munshiganj & Total \\
Electricity & & & \\
$\quad$ Electricity supply & 71.5 & 76.5 & 73.0 \\
$\quad$ Water system & & & \\
$\quad$ Own sources of supply & 61.8 & 76.5 & 66.1 \\
$\quad$ Bring water from elsewhere & 38.2 & 23.5 & $33.9 *$ \\
Mode of communication & & & \\
$\quad$ Land phone/Mobile phone & 0.0 & 9.8 & $2.9 * *$ \\
$\quad$ Laptop computer & 17.1 & 35.3 & $22.4 * *$ \\
N & 123 & 51 & 174 \\
\hline
\end{tabular}

*Significant at 0.05 level. **Significant at 0.01 level. ***Significant at 0.001 level.

In Chittagong district, none of the UHFWCs had a communication system with land phone or dedicated mobile phone while phone communication was found in 10 percent of UHFWCs in Munshiganj district. Laptop computer was found in close to one-fourth of the assessed UHFWCs, with large difference between intervention districts (Munshiganj: 35\%, Chittagong: 17\%) (Table 3).

Not all UHFWCs had a signboard; one-fourth were yet to have a signboard. Similarly, approximately 20 percent of the facilities needed a sign announcing that maternal, newborn, and child health services are available at the UHFWC (not shown).

Table 4 illustrates the sanitation situation of UHFWCs. It was found that 79-85 percent of the facilities had usable toilets. On an average, there were three toilets in a UHFWC and 60-76 percent of the UHFWCs had water supply in toilets. Only one in five UHFWCs had separate toilets for males and females (Chittagong: 25\%, Munshiganj: 18\%). Toilets were clean in 33 percent of the facilities, with large difference between districts (Munshiganj: 48\%, Chittagong: $27 \%)$. 
Table 4: Condition of toilets in Union Health and Family Welfare Centers (percent)

\begin{tabular}{lrrr}
\hline Observation items & Chittagong & Munshiganj & Total \\
Availability of usable toilets & 84.6 & 78.8 & 82.8 \\
N & 123 & 51 & 174 \\
Separate toilets for male and female & 25.0 & 17.5 & 22.9 \\
Water supply in toilets & 76.0 & 60.0 & $71.5^{*}$ \\
Cleanliness of toilets & 26.9 & 47.5 & $32.6^{* *}$ \\
Soap in toilets & 28.9 & 20.0 & 26.4 \\
N & 104 & 40 & 144 \\
Number of toilets per facility (mean) & 2.96 & 2.88 & 2.94 \\
\hline
\end{tabular}

*Significant at 0.05 level. **Significant at 0.01 level. *** Significant at 0.001 level.

Table 5 shows the physical condition of waiting space and FWV room of the Union Health and Family Welfare Center. Nine in every ten facilities had adequate light in both waiting space and FWV room. A small number of the facilities had water supply in both rooms. It has been observed that water supply in the waiting room was available in only 13 percent of facilities while it was 28-40 percent in case of FWV room. Two-thirds of the facilities had a toilet in the waiting space (Chittagong: 68\%, Munshiganj: 60\%) and 51-58 percent had toilets in FWV room. There was electric fan at the waiting space in only 11-17 percent of the facilities while it was as high as 70 percent for FWV room. Three in every four facilities were found with clean floor in waiting space and FWV room. Almost 90 percent of UHFWCs had door with lock in the FWV room, yet more than 40 percent were to ensure visual privacy at the FWV room.

Table 5: Physical condition of waiting and FWV rooms in Union Health and Family Welfare Centers (percent)

\begin{tabular}{lrr|rr}
\hline Observation items & \multicolumn{2}{c|}{$\begin{array}{c}\text { Waiting room } \\
\text { Chittagong }\end{array}$} & $\begin{array}{r}\text { Munshiganj } \\
\text { Chittagong }\end{array}$ & $\begin{array}{c}\text { Munshiganj } \\
\text { Amenities }\end{array}$ \\
$\quad$ & & & & \\
$\quad$ Adequate light & 87.5 & 93.8 & 90.5 & 95.8 \\
$\quad$ Water supply & 12.5 & $12.5^{*}$ & 28.4 & $39.6^{*}$ \\
$\quad$ Toilet & 68.3 & 60.4 & 50.9 & 58.3 \\
$\quad$ Clean floor & 77.5 & 77.1 & 81.9 & 75.0 \\
$\quad$ Electric fan & 10.8 & 16.7 & 70.7 & 68.8 \\
$\quad$ Chair/bench & 82.5 & 83.3 & 93.1 & 85.4 \\
Privacy & & & & \\
$\quad$ Door with lock & NA & NA & 84.5 & 87.5 \\
$\quad$ Visual privacy and window with & NA & NA & 58.6 & 52.1 \\
$\quad$ curtain & & & & \\
N & 120 & 48 & 116 & 48 \\
\hline
\end{tabular}

*Significant at 0.05 level. **Significant at 0.01 level. ***Significant at 0.001 level. 


\section{LABOR ROOM}

The availability of a labor room at the UHFWC is almost universal and nearly 90 percent were in good working condition. Yet, in 10 percent of UHFWCs, labor rooms were not in usable condition. In regard to recovery rooms, 18-29 percent of the UHFWCs did not have one and another 6-8 percent were not usable (Figure 2).

Figure 2: Labor and recovery rooms in Union Health and Family Welfare Centers (percent)

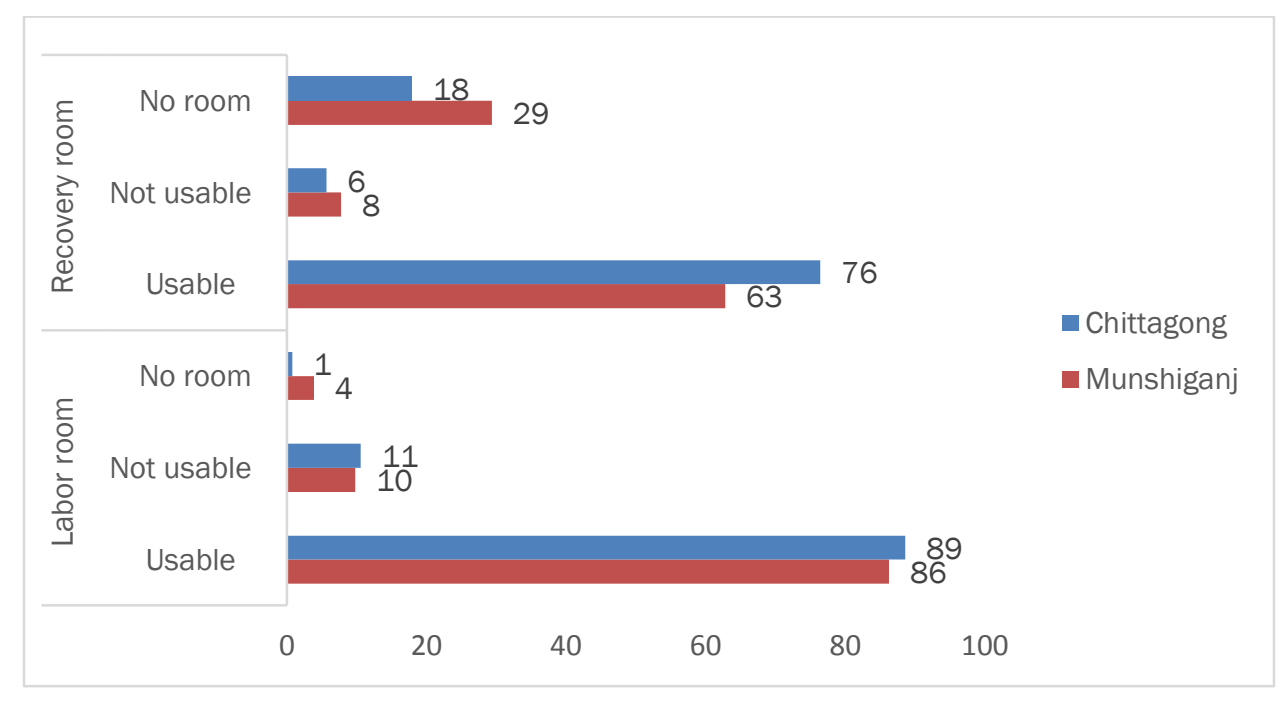

Amenities in labor rooms across the study facilities did not vary. In both districts, half of the facilities had toilets in labor room. The major problem for the UHFWC lies with a 24hour supply of electricity; only 22 percent of facilities reported using a generator as an alternate source of electricity in Chittagong and only 7 percent in Munshiganj. Similarly, water supply in the labor room was found in a smaller number of facilities (37\% and 35\%, in Chittagong and Munshiganj, respectively). While 80 percent of facilities had door with lock in the labor room, visual privacy was not adequate for labor rooms, where less than half had visual privacy. Overall, delivery rooms were not clinically well-equipped. The assessment reveals a shortage of operating lights and a functional operating table in the labor room, and large gaps between districts were found. More UHFWCs in Chittagong than in Munshiganj had delivery/operating table in working condition (85\% and $70 \%$ respectively) (Table 6). 
Table 6: Situation of the labor room in Union Health and Family Welfare Centers (percent)

\begin{tabular}{lrrr}
\hline Item & Chittagong & Munshiganj & Total \\
Physical & & & \\
$\quad$ Workable generator & 22.0 & 7.0 & 17.8 \\
Water supply & 36.7 & 34.9 & 36.2 \\
$\quad$ Toilet & 56.9 & 51.2 & 55.3 \\
$\quad$ Electric fan & 51.4 & 48.8 & 50.7 \\
$\quad$ Door with lock & 79.8 & 79.1 & 79.6 \\
$\quad$ Visual privacy and window with curtain & 43.1 & 48.8 & 44.7 \\
\hline Clinical & & & \\
$\quad$ Has fixed or portable operating light & 63.3 & $32.6 * * *$ & 54.6 \\
$\quad$ Functional delivery/operating table & 85.3 & $69.8^{*}$ & 80.9 \\
\hline $\mathbf{N}$ & 109 & 43 & 152 \\
\hline
\end{tabular}

*Significant at 0.05 level. **Significant at 0.01 level. ***Significant at 0.001 level.

The assessment found a discouraging situation of recovery rooms at the UHFWC.

Differences between two districts were negligible. Less than half of the facilities had a bed in recovery room (Chittagong: 44\%, Munshiganj: 41\%). Chairs and electric fans were available in one-fourth of the facilities (not shown).

\section{B. Human Resources}

The assessment reveals almost full availability of FWVs in Chittagong (94\%), whereas 18 percent of the facilities in Munshiganj reported non-availability of FWVs. On the other hand, the availability of SACMO is almost universal in Munshiganj (96\%), while it is notably low at 78 percent in Chittagong.

Regarding indirect providers and support staff, however, the situation is mixed. A shortage of pharmacists was found as the major problem with health workforce at UHFWCs. Less than 20 percent of UHFWCs reported the availability of pharmacists. Nearly all facilities in Munshiganj had an aya available while it is notably low at 73 percent in Chittagong (Figure 3). 
Figure 3: Staff composition at the Union Health and Family Welfare Center (percent)

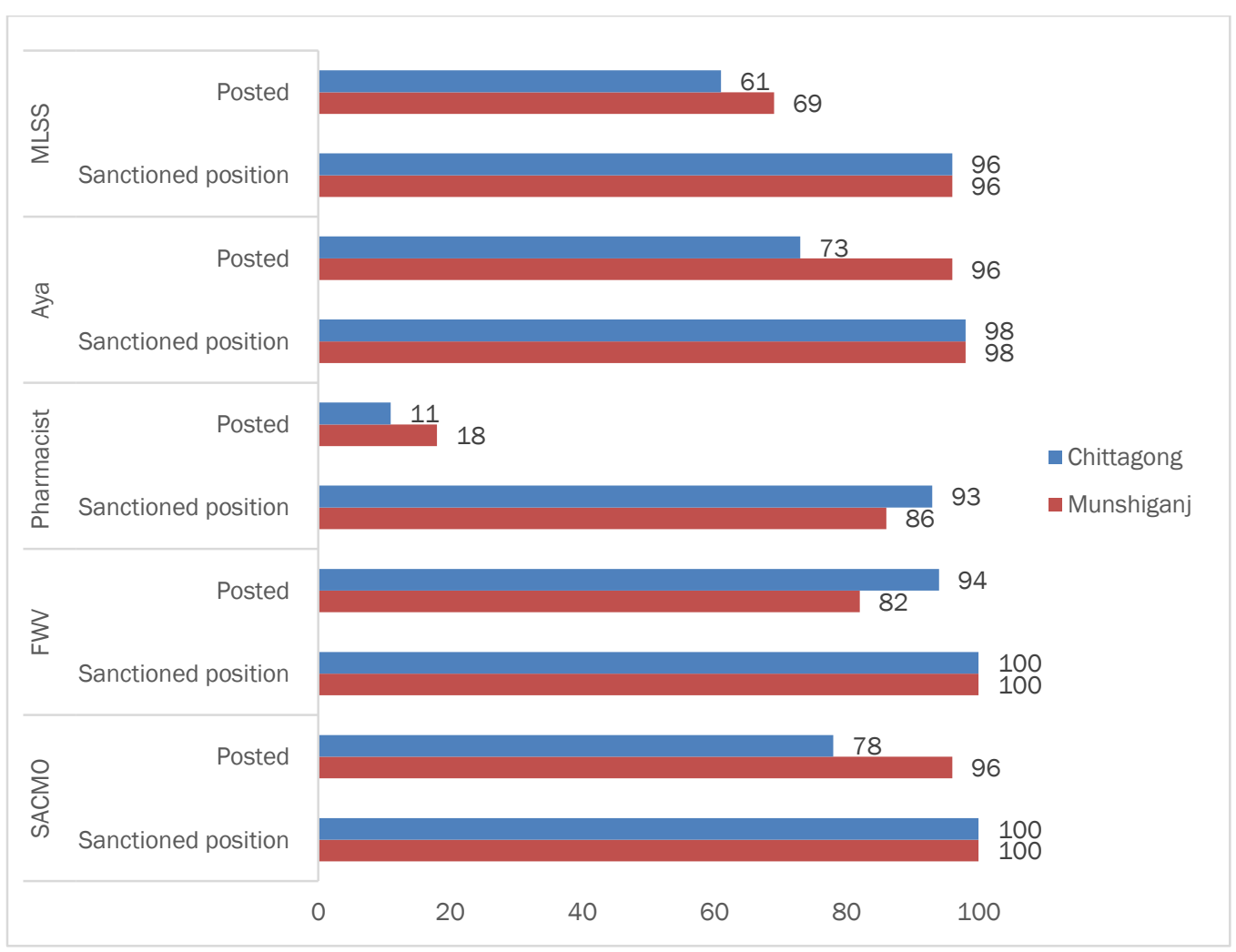

MLSS $=$ Member of Lower Subordinate Services. FWV = Family Welfare Visitor.

$\mathrm{SACMO}=$ Sub-Assistant Community Medical Officer.

Table 7 presents a range of training received in the past five years by the FWVs responsible for providing delivery services at the UHFWC. Overall, half of the FWVs received training on midwifery and one-fourth on the active management of the third stage of labor. While more than three-fourths of FWVs were trained on menstrual regulation (MR), a small proportion received training on post abortion care (Chittagong: $6 \%$, Munshiganj: 24\%). Among other clinical trainings, 40 percent of FWVs were trained on infection prevention (e.g., instrument processing), while less than 10 percent received training on waste management. Opportunity to receive management training is very limited. Ten percent of FWVs or less had training on record keeping, and drugs and supplies management. 
Table 7: Training received by Family Welfare Visitors (percent)

\begin{tabular}{lrrr}
\hline Training & Chittagong & Munshiganj & Total \\
Services $†$ & & & \\
$\quad$ Midwifery & 55.9 & 52.6 & 54.7 \\
$\quad$ Active management of third stage of labor & 26.3 & 18.4 & 24.3 \\
$\quad$ Menstrual regulation & 76.2 & 81.6 & 77.5 \\
$\quad$ Post-abortion care & 5.9 & $23.7 * * *$ & 10.2 \\
$\quad$ Integrated management of childhood illnesses & 10.2 & $31.6 * * *$ & 15.3 \\
$\quad$ Infection prevention & & & \\
$\quad$ Sterilization through instrument processing & 38.1 & 42.1 & 39.1 \\
$\quad$ Waste management & 9.3 & 7.8 & 8.9 \\
$\quad$ Management & & & \\
$\quad$ Drugs and supplies management & 1.7 & $7.9 *$ & 3.2 \\
$\quad$ Record keeping & 9.3 & 13.2 & 10.2 \\
$\mathrm{~N}$ & 118 & 38 & 156 \\
\hline
\end{tabular}

†Multiple responses. * Significant at 0.05 level. **Significant at 0.01 level. ***Significant at 0.001 level.

To ensure regularity of services, residential quarters for staff have been constructed at the UHFWCs. Nearly all facilities (98\%) in Chittagong had residential quarters for service providers, while 12 percent in Munshiganj had no residential quarters. The majority of service providers did not live on the premises. More service providers (FWVs and SACMOs) in Munshiganj than in Chittagong were residential. FWVs in Munshiganj district were twice as likely to live inside the compound as those from Chittagong district (49\%, $23 \%$ respectively). The gap between two districts in residential status of SACMO was even larger (Chittagong: 23\%, Munshiganj: 64\%) (Figure 4).

Figure 4: Accommodation and residential status of service providers (percent)

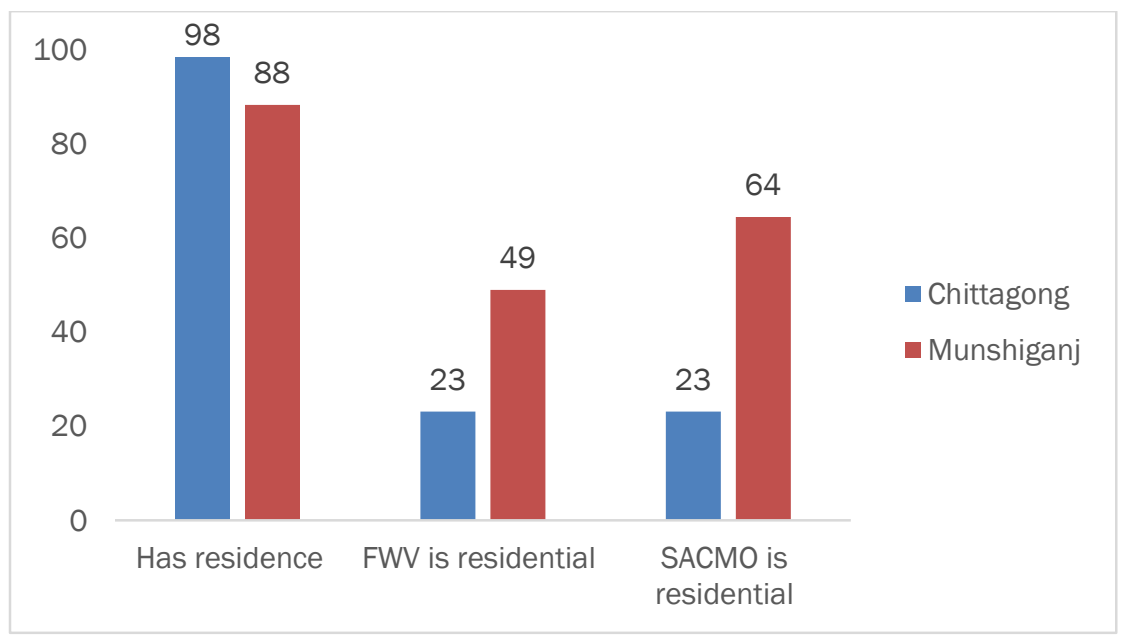

FWV $=$ Family Welfare Visitor. SACMO $=$ Sub-Assistant Community Medical Officer . 


\section{Equipment, Logistics, and Supplies}

Figure 5 shows the availability of drugs and dietary supplement (DDS) kits for the UHFWC in the month preceding the assessment. A timely supply of one DDS kit per month was more common in Munshiganj than in Chittagong ( $76 \%$ vs. $58 \%$ ). More facilities in Chittagong than in Munshiganj reported receiving two or more kits, which may be accumulated for more than one month. Yet in both districts, 10 percent of facilities did not receive any DDS kits, suggestive of delayed supply of the kits. None of the facilities received "safe delivery kits" at the time of facility assessment (not shown). The DGFP has recently introduced safe delivery kits for its UHFWCs.

Figure 5: Supply of DDS kits in the previous month (percent)

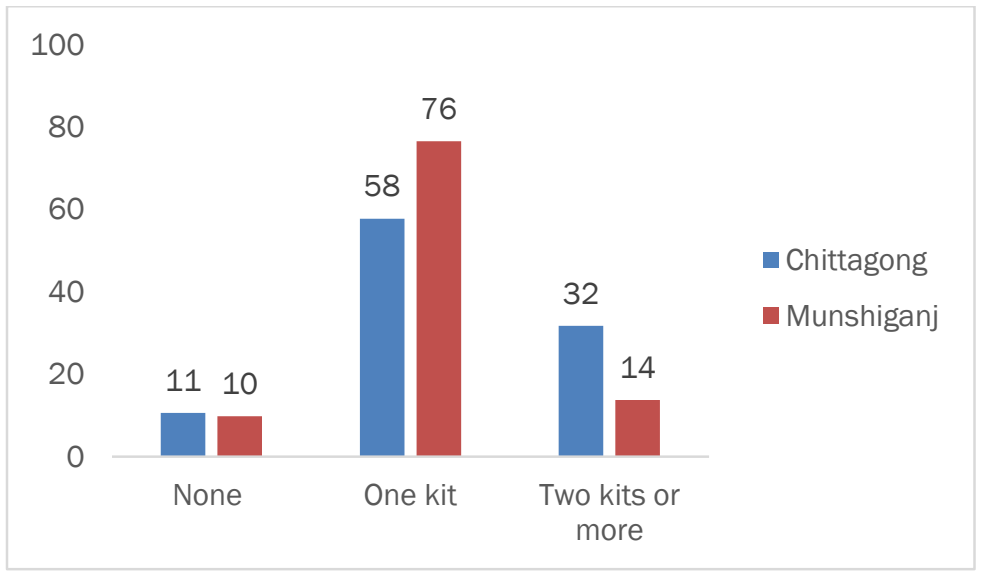

Behavior change communication (BCC) materials, such as flipchart and brochure, are used by providers for counseling. The majority of UHFWCs were without these materials and the availability of flipchart or brochure on any of the services on the day of assessment did not exceed 40 percent. For example, flipchart or brochure on maternal health services, e.g., antenatal care, postnatal care and delivery services were available at 27 to 40 percent of UHFWCs. The assessment also found limited availability of communication materials on delivery and danger signs of pregnancy, with large gap between districts (Munshiganj: 39\%, Chittagong: 21\%) (not shown).

For mass viewing, the availability of BCC materials such as posters and wall writings at the UHFWC was mixed. In most cases, differences between districts are large. More than 80 percent of UHFWCs in Munshiganj had poster and wall writing on citizen's charter compared with 62 percent in Chittagong. More facilities in Munshiganj than Chittagong had posters and wall writings on delivery and danger signs of pregnancy while a reverse condition is noticed on the availability of posters/writings on newborn care and immunization, yet with a maximum availability of only 56 percent (Figure 6). 
Figure 6: Availability of poster or wall writing for mass viewing (percent)

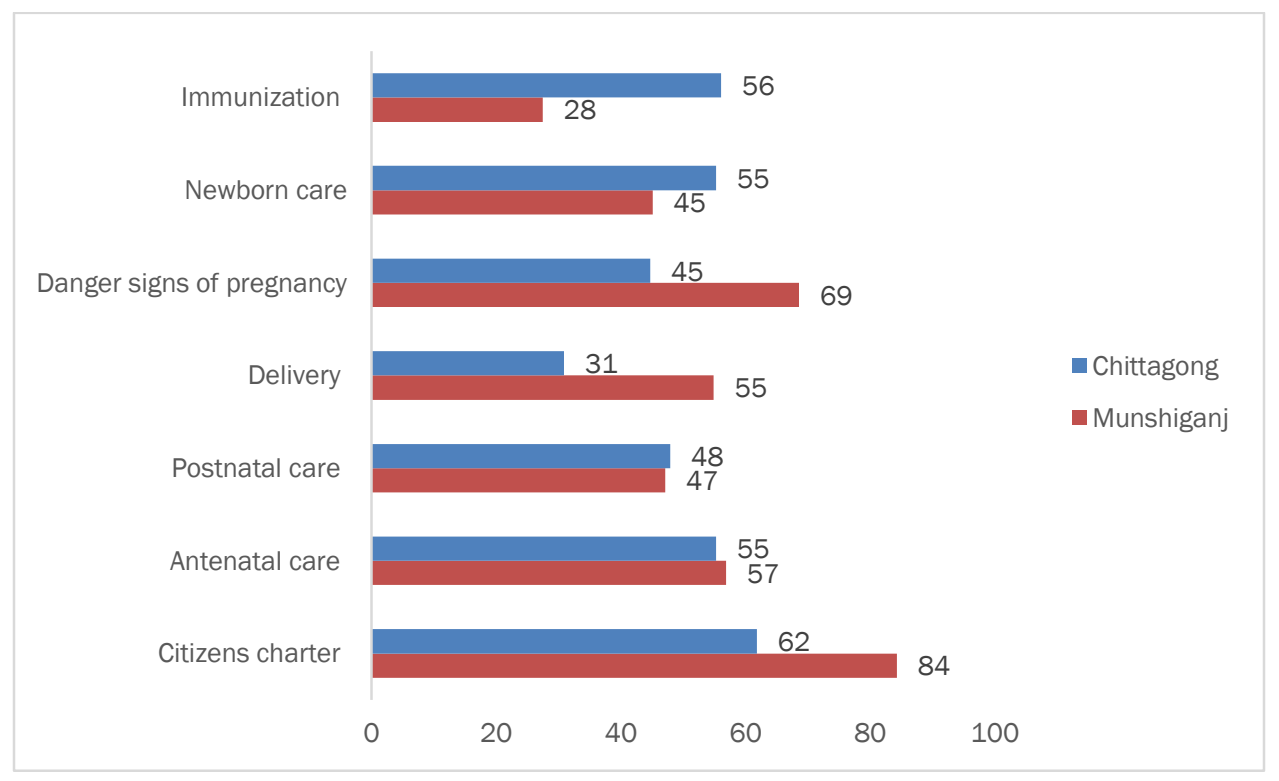

\section{Service Delivery}

\section{NORMAL DELIVERY SERVICES}

Not all UHFWCs provide normal delivery services. More facilities in Chittagong were found to provide normal delivery services than in Munshiganj (72\% and 59\%, respectively) (not shown). The assessment found a lack of trained providers (Chittagong: 49\%, Munshiganj: $43 \%$ ), equipment and supplies (31\% and $43 \%$, respectively), and supply of water and electricity (3\% and 5\%, respectively) as supply-side reasons for not providing normal delivery services (Figure 7 ).

Figure 7: Reasons for not providing normal delivery services (percent)

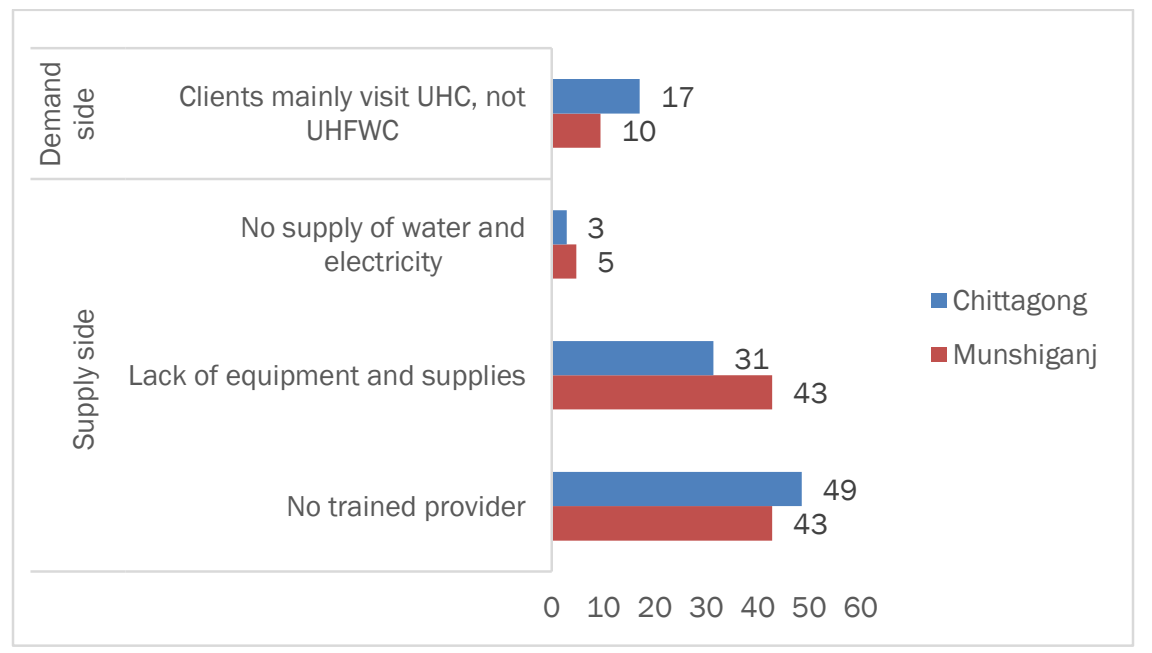

$\mathrm{UHC}=$ Upazila Health Complex. UHFWC= Union Health and Family Welfare Center 
There was no clinical provision for Essential Newborn Care (ENC) at the UHFWC. Service providers were not trained to provide clinical ENC. None of the facilities had basic equipment for ENC, e.g., pulse oximeter, airway-suction equipment, and an oxygen cylinder (not shown).

\section{REFERRAL}

As shown in Figure 8, service providers refer emergency clients mainly to government facilities. The Upazila Health Complex is the facility where a large proportion of referral cases are sent (67-86\%), and the District Hospital is the next major facility where referral cases are sent (23-33\%).The proportion of clients referred to the Upazila Health Complex is much higher in Chittagong than in Munshiganj. Nonuse of the Mother and Child Welfare Center (MCWC) as a referral facility is evident in Chittagong, while in Munshiganj 18 percent of complicated cases were referred to MCWCs. Yet, 22 percent of providers sent referral cases to private providers in Munshiganj while such referrals were nearly absent in Chittagong.

Figure 8: Clients referred to higher-level facilities from the UHFWC (percent)*

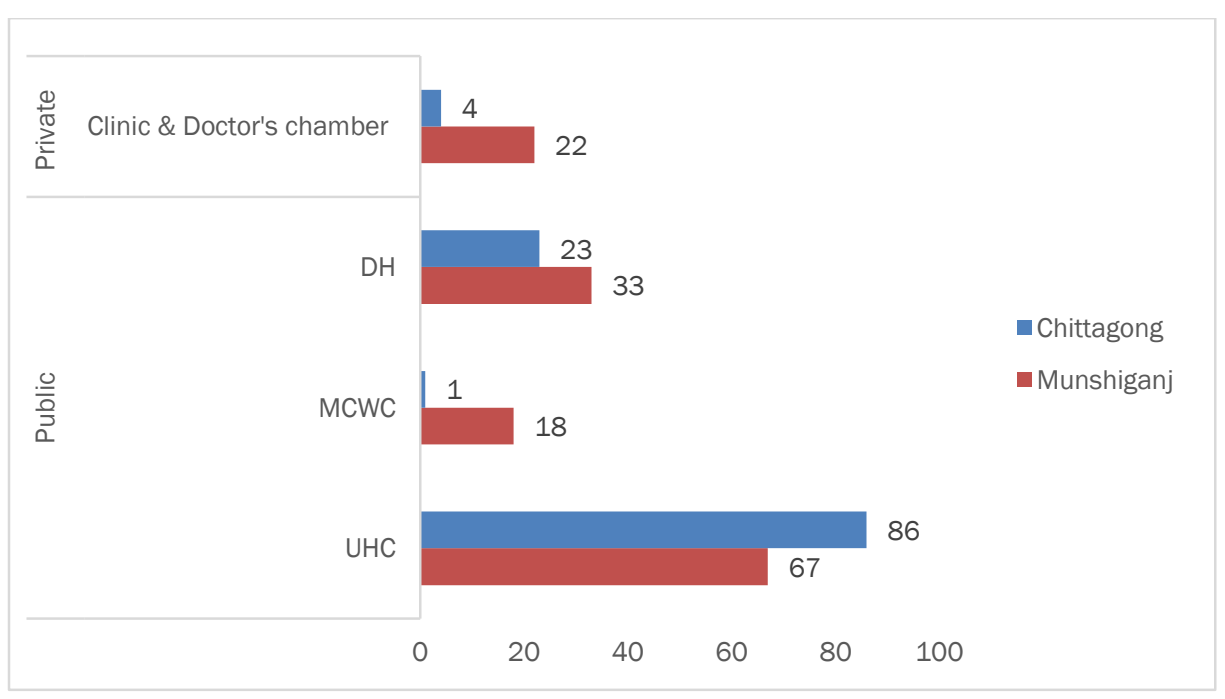

*Multiple responses. $\mathrm{DH}=$ District Hospital. $\mathrm{MCWC}=$ Mother and Child Welfare Center. $\mathrm{UHC}=$ Upazila Health Complex.

\section{INFECTION PREVENTION}

Figure 9 depicts overall management of infection prevention in terms of instrument processing and waste management. In both districts, instruments were sterilized mainly through boiling (Chittagong: $84 \%$, Munshiganj: $75 \%$ ). Chlorination is the next most widely used process for sterilization and facilities in Chittagong used these processes more often than in Munshiganj (76\% and 35\% respectively). Both districts witnessed a low use of autoclave. 
In both districts, use of separate bins for liquid, solid, and sharp items for waste management is nearly universal (Chittagong: 96\%; Munshiganj: 92\%). However, waste management through chlorinated bins was not commonly practiced in Munshiganj (35\%). It is nearly universal in both districts to bury perishable materials as part of waste management (Chittagong: 92\%; Munshiganj: 84\%). Use of incinerator to burn waste materials was 65 percent in Chittagong compared with 49 percent in Munshiganj (Figure 9).

Figure 9: Practice of sterilization of equipment and waste management (percent)
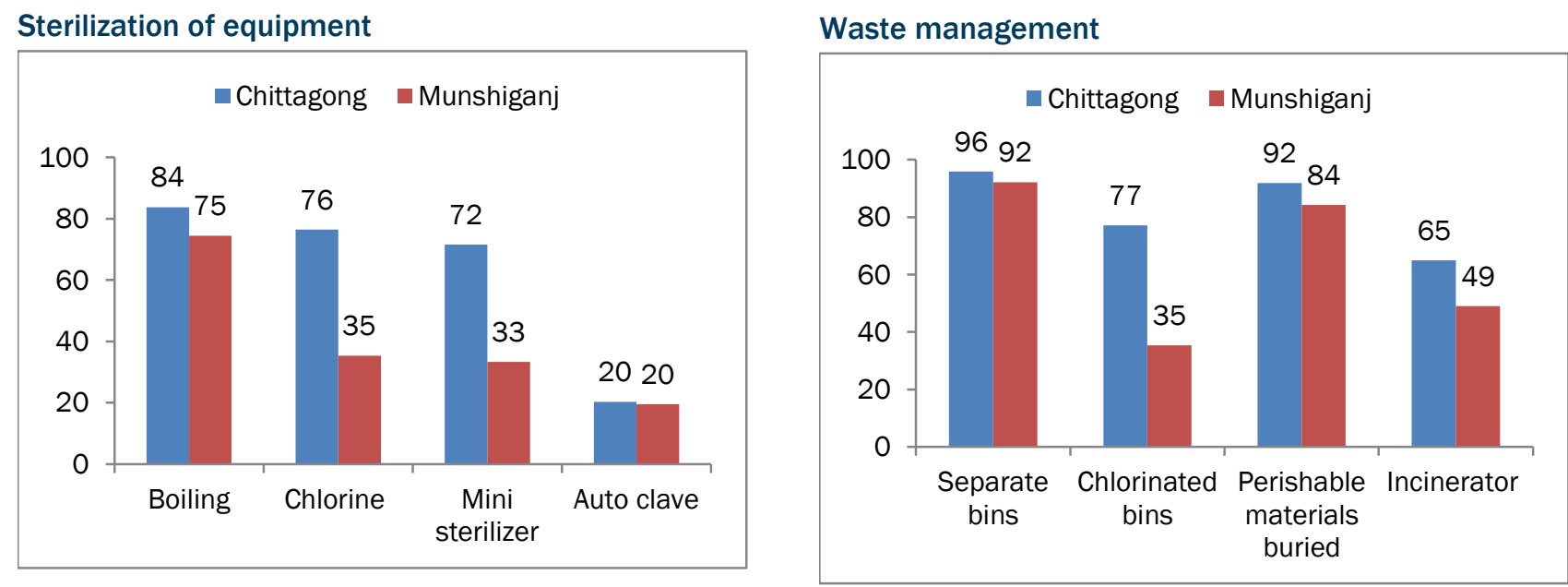

\section{E. Management}

Table 8 shows the management of UHFWCs. Nearly all UHFWCs in both districts had separate store rooms and these facilities stored stocks by expiration dates. It is encouraging to note that all facilities reported updating the stock of drugs and supplies in compliance with monthly reporting. Although all UHFWCs are required to update the stocks monthly, 68 percent of the facilities updated stocks of supplies on a daily basis, with 81 percent of facilities in Chittagong updating stocks of supplies on a daily basis.

The practice of submitting monthly performance reports on family planning, maternal health and general health was universal in both the districts. Almost all the facilities in both districts submit all monthly progress reports except immunization which is 33 percent in Munshiganj and 48 percent in Chittagong.

Not all facilities had the required service protocols on the day of assessment. Among all protocols, the UHFWC operating manual was most frequently available at UHFWCs, with notable gaps between districts (Munshiganj: 84\%, Chittagong 66\%). The family planning manual is the next most commonly available protocol in both the districts (Munshiganj: $65 \%$, Chittagong 63\%). Yet, one-third of the facilities did not have the UHFWC Operating Manual and Family Planning Manual, and the protocol on infection prevention was found in only 35 percent of the UHFWCs. The assessment found greater availability of standard protocols at UHFWCs in Munshiganj than in Chittagong (Table 8). 
Table 8: Management of Union Health and Family Welfare Centers (percent)

\begin{tabular}{lrrr}
\hline Indicator & Chittagong & Munshiganj & Total \\
$\begin{array}{l}\text { Separate store room in facilities for } \\
\text { supplies/drugs }\end{array}$ & 98.4 & 94.1 & 97.1 \\
N & 123 & 51 & 174 \\
Stocks management & & & \\
Stocks are stored by expiration dates & 100.0 & 97.9 & 99.4 \\
Update stocks of supply and drugs & & & \\
$\quad$ Daily & 81.0 & 33.3 & 67.5 \\
$\quad$ Weekly & 14.0 & 8.3 & 11.8 \\
$\quad$ Monthly & 5.0 & $58.4 * * *$ & 20.7 \\
N & 121 & 48 & 169 \\
Monthly reporting & & & \\
$\quad$ Family planning performance report & 99.2 & 96.1 & 98.3 \\
$\quad$ Maternal and child health report & 97.6 & 98.0 & 97.7 \\
$\quad$ Menstrual regulation report & 96.7 & $84.3^{*}$ & 93.1 \\
$\quad$ General health report & 99.2 & 100.0 & 99.4 \\
$\quad$ Immunization report & 48.0 & 33.3 & 43.7 \\
Manuals/Protocols available & & & \\
$\quad$ UHFWC operating manual & 65.9 & $84.3^{* *}$ & 71.3 \\
$\quad$ Family planning & 63.4 & 64.7 & 63.8 \\
$\quad$ Infection prevention & 29.3 & $47.1^{* * *}$ & 34.5 \\
$\quad$ Instrument processing & 32.5 & $58.8^{* * *}$ & 40.2 \\
N & 123 & 51 & 174 \\
\hline
\end{tabular}

*Significant at 0.05 level. **Significant at 0.01 level. ***Significant at 0.001 level.

Union Health and Family Planning Committees (UHFPCs) oversee the activities of UHFWCs. The assessment reveals that committees had been formed in four-fifths of the unions (Chittagong: $82 \%$, Munshiganj: $80 \%$ ) and only $10-18$ percent of those committees were found functional where monthly meetings were held, mostly irregularly. None of the committees was empowered to oversee the activities of the UHFWC and to contribute to the UHFWC maintenance (not shown).

Figure 10: Status of Union Health and Family Planning Committees (percent)

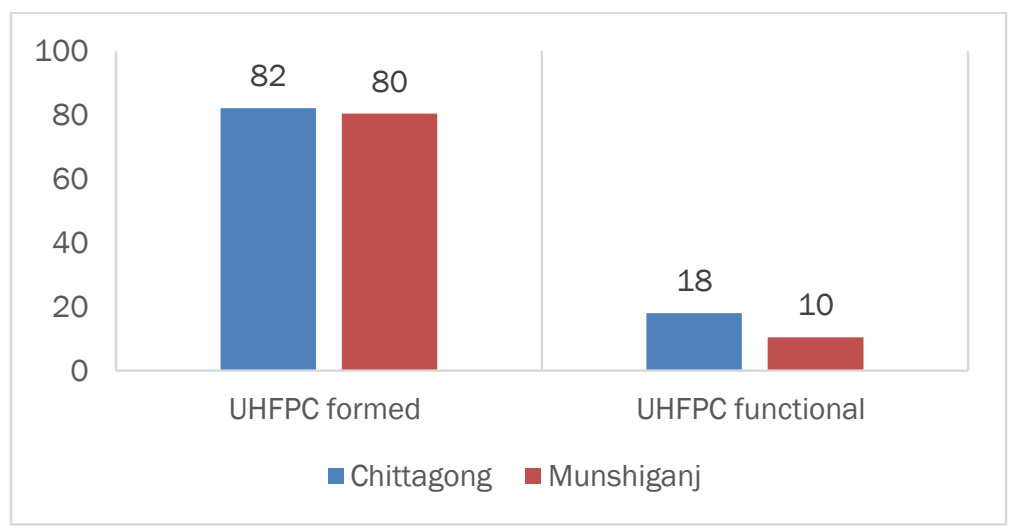

UHFPC $=$ Union Health and Family Planning Committee 


\section{FINDINGS FROM THE PROVIDER SURVEY}

This section highlights the findings from the survey with 27 Family Welfare Visitors (FWVs), focusing on their maternal health knowledge and capacities, and experience of working at the Union Health and Family Welfare Center (UHFWC) in providing 24-hour normal delivery services.

\section{A. Age, Recruitment, and Placement}

Overall, the average age of the FWVs is 44 years, with no remarkable difference between the two districts. Half of the FWVs are in their 40s and one-fourth are aged 50 years and older. Only 2 out of 27 FWVs were under age 30.

Table 9: Distribution of FWVs by age groups (number)

\begin{tabular}{lrrr} 
Age group & Chittagong & Munshiganj & Total \\
$<30$ years & 1 & 1 & 2 \\
$31-40$ years & 2 & 3 & 5 \\
$41-50$ years & 10 & 3 & 13 \\
$>50$ years & 2 & 5 & 7 \\
N & 15 & 12 & 27 \\
Mean age (years) & 42.8 & 45.9 & 44.2 \\
\hline
\end{tabular}

Interviews with the FWVs revealed that the majority of them were recruited before 1994. Of $27 \mathrm{FWVs}, 18$ had been working for more than 20 years, four had been working 5 years or less, and remaining five having a work experience of 11-20 years. More than half of the FWVs will retire by 2020 , which requires immediate attention of program managers.

Table 10: Working experience of FWVs (number)

\begin{tabular}{lrrr} 
Work experience & Chittagong & Munshiganj & Total \\
$>20$ years & 11 & 7 & 18 \\
$11-20$ years & 2 & 3 & 5 \\
$6-10$ years & 0 & 0 & 0 \\
$\leq 5$ years & 2 & 2 & 4 \\
N & 15 & 12 & 27 \\
\hline
\end{tabular}

FWVs who are posted at UHFWCs are entrusted with the responsibility to provide satellite services two days a week and thus FWVs are available at the UHFWC four days a week. In practice, two-thirds of FWVs provide services at the Satellite Clinic twice in a week while the rest provide satellite services once a week. Three-fourths of the FWVs emphasized the need for additional service providers to bridge the gap in service delivery at the UHFWC (Table 11). Regarding the status of accommodation, 11 out of 12 FWV surveyed 
in Munshiganj resided in the facility campus, while 8 out of 15 FWVs in Chittagong were residential (not shown).

Table 11: Satellite clinic services provided by FWVs (number)

\begin{tabular}{lrrr}
\hline Indicator & Chittagong & Munshiganj & Total \\
Provide services in satellite clinics & 15 & 12 & 27 \\
Frequency of services in satellite clinics & & & \\
$\quad$ Once in a week & 9 & 0 & 9 \\
$\quad$ Twice in a week & 6 & 12 & 18 \\
Additional service provider is needed & 11 & 10 & 21 \\
N & 15 & 12 & 27 \\
\hline
\end{tabular}

\section{B. Provider Competence}

Provider competence is defined in this report as possessing sufficient knowledge and skills to comply with standard practices on maternal health care. In assessing knowledge and skills, an arbitrary composite competency score is used.

\section{COUNSELING SKILLS}

Counseling skills of FWVs on maternal health care were assessed on three broad aspects: general counseling, birth planning counseling, and advice on discharge after delivery. An arithmetic method is used for estimating the competency score for each aspect of care to obtain a summary performance of FWVs. Overall, counseling skills of FWVs was discouraging, not exceeding a score of 0.68 out of 1.00 (Table 12). The level of counseling competency is higher in Chittagong than Munshiganj. Low score on maternal health counseling can be accounted for by lack of opportunities for in-service training or absence of regular supportive supervision.

Of three aspects of counseling, "birth planning" earned highest score, where Chittagong outperformed Munshiganj (Chittagong: 0.65, Munshiganj: 0.55). In 5 of the 8 indicators on birth planning counseling, more than half of FWVs give pregnant mothers advice on selecting skilled birth attendant, selecting facility for emergency, identifying blood group and managing donor, arranging money in case of emergency, and arranging transportation in case of emergency. Only one-third of the providers give advice on arranging necessary supplies in case of home delivery. FWVs should provide adequate information on birth planning to every pregnant woman so that the latter become well prepared before the child birth.

On general counseling, gaps in the score between districts are much greater (Chittagong: 0.68, Munshiganj: 0.46). All FWVs advised pregnant women about balanced diet and twothirds advised on avoiding heavy work and taking proper rest. Alarmingly, only 2 out of 27 FWVs provided information to pregnant women on danger signs of pregnancy. The lowest score is on counseling at "discharge after delivery" as earned by FWVs, with a large variation between districts (Chittagong: 0.57 , Munshiganj: 0.38 ). Immunization of 
baby (Chittagong: 15 out of 15, Munshiganj: 6 out of 12) and correct method of breastfeeding (Chittagong: 14 out of 15, Munshiganj: 7 out of 12) were the most common advice given at the time of discharge of new mothers. In contrast, less than half of FWVs counseled new mothers on nutrition, uptake of vitamin-A capsule, and accepting family planning methods.

Table 12: Counseling skills of FWVs on maternal health services (number)

\begin{tabular}{|c|c|c|c|}
\hline Maternal health counseling* & Chittagong & Munshiganj & Total \\
\hline \multicolumn{4}{|l|}{ General counseling } \\
\hline Advise mother about balanced diet & 15 & 12 & 27 \\
\hline Inform about danger signs of pregnancy & 1 & 1 & 2 \\
\hline Avoiding heavy work & 14 & 7 & 21 \\
\hline Rest during pregnancy & 14 & 6 & 20 \\
\hline TT injection & 8 & 4 & 12 \\
\hline Inform follow-up & 9 & 3 & 12 \\
\hline Composite score & 0.68 & 0.46 & 0.58 \\
\hline \multicolumn{4}{|l|}{ Birth planning } \\
\hline Select skilled birth attendant & 4 & 11 & 15 \\
\hline Select facility for delivery & 7 & 6 & 13 \\
\hline Select facility for emergency & 10 & 5 & 15 \\
\hline Arrange money in case of emergency & 15 & 10 & 25 \\
\hline Arrange transportation in case of emergency/delivery & 14 & 10 & 24 \\
\hline $\begin{array}{l}\text { Arrange somebody who can accompany during } \\
\text { emergency/companion during delivery }\end{array}$ & 11 & 1 & 12 \\
\hline Collect necessary supplies in case of home delivery & 5 & 4 & 9 \\
\hline Identify blood group and manage donor & 12 & 6 & 18 \\
\hline Composite score & 0.65 & 0.55 & 0.61 \\
\hline \multicolumn{4}{|l|}{ Advice on discharge after delivery } \\
\hline Receiving postnatal care & 8 & 2 & 10 \\
\hline Immunization of baby & 15 & 6 & 21 \\
\hline Correct method of breastfeeding & 14 & 7 & 21 \\
\hline Uptake of vitamin-A capsule & 3 & 5 & 8 \\
\hline Mother's nutrition & 8 & 3 & 11 \\
\hline Family planning & 3 & 4 & 7 \\
\hline Composite score & 0.57 & 0.38 & 0.48 \\
\hline$N$ & 15 & 12 & 27 \\
\hline
\end{tabular}

* Multiple responses. TT=Tetanus Toxoid. 


\section{KNOWLEDGE ON PREGNANCY CARE, DELIVERY COMPLICATIONS, AND NEWBORN CARE}

\section{Awareness of basic check-ups during pregnancy}

Service providers were asked about the examinations they perform to ensure pregnancy of a woman. All FWVs mentioned using the last menstrual period (LMP) of a woman to determine pregnancy and two-thirds reported using urine test. A few FWVs perform abdominal examination for determining the pregnancy of a woman (Table 13).

Table 13: Examinations performed by FWVs to ensure pregnancy (number)

\begin{tabular}{lrrr}
\hline Pregnancy examinations & Chittagong & Munshiganj & Total \\
Last menstrual period & 15 & 12 & 27 \\
Pregnancy test (urine test) & 7 & 12 & 19 \\
Abdomen examination & 4 & 2 & 6 \\
N & 15 & 12 & 27 \\
\hline
\end{tabular}

Table 14 shows the extent of knowledge on the examinations needed by pregnant women during the first pregnancy check-up. All 27 FWVs mentioned measurement of blood pressure and 26 mentioned measuring weight. Yet, nearly half of FWVs do not measure the height of pregnant women. Overall, the level of knowledge on required physical examinations for the first antenatal care visit was higher in Chittagong than in Munshiganj (Chittagong: 0.87 out of 1.00 , Munshiganj: 0.73 out of 1.00 ).

Table 14: Knowledge of FWVs on the types of examinations a woman needs during first antenatal care visit (number)

\begin{tabular}{lrrr}
\hline Types of examinations* & Chittagong & Munshiganj & Total \\
Physical & & & \\
Height measure & 11 & 4 & 15 \\
Weight measure & 15 & 11 & 26 \\
Blood pressure measure & 15 & 12 & 27 \\
Uterine height & 11 & 8 & 19 \\
Composite score & 0.87 & 0.73 & 0.81 \\
Laboratory & & & \\
Hemoglobin & 10 & 6 & 16 \\
Sugar & 4 & 6 & 10 \\
Blood group & 8 & 7 & 15 \\
Albumin & 9 & 8 & 17 \\
Ultra-sonogram & 2 & 4 & 6 \\
$\quad$ Composite score & 0.44 & 0.52 & 0.47 \\
N & 15 & 12 & $\mathbf{2 7}$ \\
\hline *Multiple responses & & &
\end{tabular}


Unlike the physical examination, the competency score on awareness of laboratory services required during prenatal checkups is low among FWVs (Chittagong: 0.44, Munshiganj: 0.52). Out of five laboratory tests required for the prenatal check-up, more than half mentioned hemoglobin, blood group, and albumin test (Table 14).

\section{Awareness of maternal health complications}

Findings indicate that FWVs had a moderate level of awareness on pregnancy and delivery complications. In three stages of complications (during pregnancy, during delivery, and after delivery), providers managed to score a maximum on complications during pregnancy (0.61-0.69) while poor knowledge on complications after delivery was evident (Chittagong: 0.54, Munshiganj: 0.40). The number of providers reporting awareness is greater in Chittagong than in Munshiganj for most of the complications.

Regarding the possible complications during pregnancy or antenatal period, out of 27 FWVs, 20 or more mentioned high blood pressure, excessive vaginal bleeding, and severe headache. Less than half of the FWVs were aware of pre-eclampsia as possible complications during pregnancy, which is a cause for concern as one-fifth of maternal deaths are due to eclampsia.

Table 15: Knowledge of FWVs on possible complications during pregnancy, during delivery, and after delivery (number)

\begin{tabular}{lcc|cc|cc}
\hline Types of complications* & \multicolumn{2}{c|}{$\begin{array}{c}\text { During pregnancy } \\
\text { Chittagong }\end{array}$} & $\begin{array}{c}\text { Dunshiganj } \\
\text { Chittagong }\end{array}$ & Munshiganj & \multicolumn{2}{c}{$\begin{array}{c}\text { After delivery } \\
\text { Chittagong }\end{array}$} \\
Severe headache & 13 & 8 & - & - & 3 & 1 \\
Blurry vision & 13 & 6 & - & - & 3 & 1 \\
High blood pressure & 14 & 9 & 14 & 9 & 12 & 3 \\
Pre-eclampsia & 7 & 4 & 6 & 1 & - & - \\
Convulsion/eclampsia & 7 & 9 & 7 & 11 & 9 & 9 \\
Excessive vaginal bleeding & 9 & 11 & 13 & 10 & 15 & 11 \\
High fever & 10 & 7 & 4 & 3 & 11 & 7 \\
Swelling feet & 10 & 5 & 7 & 3 & 4 & 2 \\
Excessive water leaking & - & - & 6 & 6 & - & - \\
Baby's hand and feet came & - & - & 7 & 5 & - & - \\
first & - & - & 13 & 6 & - & - \\
Prolonged labor & - & - & 10 & 8 & - & - \\
Obstructed labor & - & - & 6 & 4 & - & - \\
Retained placenta & - & - & 1 & 3 & - & - \\
Ruptured uterus & 15 & 12 & 15 & 12 & 15 & 12 \\
$\mathrm{~N}$ & 0.69 & 0.61 & 0.52 & 0.48 & 0.54 & 0.40 \\
Composite score & & & & \\
\hline
\end{tabular}

*Multiple responses

On the awareness of complications during delivery, the score is almost identical in the two study districts (Chittagong: 0.52, Munshiganj: 0.48). Among the delivery complications, high blood pressure and excessive vaginal bleeding were widely known to 
FWVs. It is alarming to note that only one-fourth of FWVs were aware of pre-eclampsia as a delivery complication. Yet, one-third are not aware of obstructed or prolonged labor, which is another major direct cause of maternal death.

Regarding the complications during post-partum period, it is encouraging to note that awareness on excessive vaginal bleeding, which is a dominant direct cause of maternal deaths, was nearly universal among FWVs. Conversely, FWVs were less aware of swelling of feet as a post-partum complication. More FWVs from Chittagong than Munshiganj were aware of all complications except convulsion (Table 15).

Overall knowledge of FWVs on the five danger signs of pregnancy was adequate. Encouragingly, FWVs in Munshiganj earned nearly an absolute score on the danger signs of pregnancy (0.98 out of 1.00). Four out of five danger signs of pregnancy were known to almost all the FWVs. However, prolonged labor as a danger sign of pregnancy was known to two-thirds of FWVs, with notable difference between study districts (Table 16). Awareness of FWVs on dangers of prolonged and obstructed labor is critical as many women experience prolonged or obstructed labor, which is another major direct cause of maternal deaths and those who survive suffer from illnesses and disabilities, such as fistula and uterine prolapse.

Table 16: Knowledge of FWVs on five danger signs of pregnancy (number)

\begin{tabular}{lrcr}
\hline Danger signs* & Chittagong & Munshiganj & Total \\
Vaginal bleeding & 15 & 12 & 27 \\
Convulsion & 13 & 12 & 25 \\
Severe headache and blurred vision & 15 & 12 & 27 \\
High fever & 14 & 12 & 26 \\
Prolonged labor & 8 & 11 & 19 \\
N & 15 & 12 & 27 \\
Composite score & 0.87 & 0.98 & 0.92 \\
\hline
\end{tabular}

*Multiple responses

\section{Awareness of neonatal complications}

Assessment of awareness of signs and symptoms of infection of newborns indicates discouraging diagnosis skills of FWVs on newborn complications. A maximum composite score of 0.50 (out of 1.00) indicates a serious deficiency in knowledge of basic neonatal care (Table 17). Four in five providers mentioned sepsis in cord and more than half mentioned difficulty in breathing and swollen eyes. Less than half of FWVs were able to mention hypothermia or hyperthermia, septic spots/boils on body, jaundice at birth, and inadequate breastfeeding /feeding as neonatal health complications. 
Table 17: Knowledge of FWVs on signs and symptoms of newborn complications (number)

\begin{tabular}{lrrr}
\hline Signs and symptoms* & Chittagong & Munshiganj & Total \\
Poor or no breastfeeding /feeding & 9 & 2 & 11 \\
Difficulty in breathing & 8 & 8 & 16 \\
Swollen eyes & 6 & 9 & 15 \\
Hypothermia or hyperthermia & 6 & 4 & 10 \\
Septic spots/Boils on body & 6 & 5 & 11 \\
Jaundice at birth & 6 & 5 & 11 \\
Convulsion & 2 & 4 & 6 \\
Sepsis in cord & 11 & 11 & 22 \\
N & 15 & 12 & 27 \\
Composite score & 0.45 & 0.50 & 0.47 \\
\hline
\end{tabular}

*Multiple responses

Compared to the awareness score on signs and symptoms of neonatal complications, the score of the awareness on basic preventive care for newborn was higher. At least twothirds of FWVs had knowledge of 5 out of 7 preventive steps for newborn care, namely, cord care, maintaining hygiene, importance of colostrum, early initiation of breastfeeding, and exclusive breastfeeding for 6 months (Table 18).

Table 18: Awareness of FWVs on basic preventive care for newborn (number)

\begin{tabular}{lrrr}
\hline Basic preventive care for newborn* & Chittagong & Munshiganj & Total \\
Importance of colostrum & 15 & 10 & 25 \\
Early initiation of breastfeeding & 9 & 9 & 18 \\
Warmth & 8 & 5 & 13 \\
Cord care & 13 & 8 & 21 \\
Eye care & 3 & 5 & 8 \\
Exclusive breastfeeding for 6 months & 9 & 9 & 18 \\
Maintaining hygiene & 12 & 9 & 21 \\
N & 15 & 12 & 27 \\
Composite score & 0.66 & 0.66 & 0.66 \\
\hline
\end{tabular}

*Multiple responses

\section{OBSTETRIC SKILLS}

Overall, the competency of clinical skills on maternal health care was high among FWVs. Exclusion of partograph from the composite index raises the score to 0.89 out of 1.00 . Most FWVs reported possessing the skills to practice bimanual examination, injecting intravenous infusions, speculum examination, suturing episiotomy, and repairing vaginal laceration. In contrast, only 5 out of $27 \mathrm{FWV}$ s reported using partograph to manage labor (Table 19). It is critical that all the FWVs are skilled to use partograph to manage labor; otherwise, there will be risk of failure to recognize obstetric complications. 
Table 19: Clinical skills of FWVs on maternal health care (number)

\begin{tabular}{lrrr}
\hline Indicators & Chittagong & Munshiganj & Total \\
Use partograph to manage labor & 1 & 4 & 5 \\
Provide intravenous infusions & 15 & 12 & 27 \\
Suture episiotomy & 13 & 10 & 23 \\
Suture (repair) vaginal laceration & 12 & 10 & 22 \\
Perform speculum examination & 13 & 12 & 25 \\
Perform bimanual examination & 15 & 12 & 27 \\
Perform menstrual regulation & 12 & 8 & 20 \\
N & 15 & 12 & 27 \\
Composite score & 0.77 & 0.81 & 0.79 \\
Composite score without partograph & 0.89 & 0.89 & 0.89 \\
\hline
\end{tabular}

\section{MANAGEMENT OF OBSTETRIC COMPLICATIONS}

Table 20 explores FWVs' skills in referring when they detect complicated cases. It is customary for FWVs to refer clients for eclampsia, prolonged labor, and excessive bleeding before or after delivery. Other major complications that lead to referral include obstructed labor and abnormal position of the baby. Only 8 reported referral for cesarean delivery.

Table 20: Pregnancy complications reported by FWVs for referral (number)

\begin{tabular}{lrrr}
\hline Complications* & Chittagong & Munshiganj & Total \\
Pre-eclampsia & 8 & 1 & 9 \\
Eclampsia & 14 & 10 & 24 \\
Prolonged labor & 13 & 9 & 22 \\
Obstructed labor & 10 & 6 & 16 \\
Fetal distress & 5 & 1 & 6 \\
Umbilical cord delivery & 2 & 0 & 2 \\
Abnormal position of the baby & 10 & 4 & 14 \\
Excessive bleeding before or after delivery & 11 & 12 & 23 \\
Excessive water leaking & 4 & 4 & 8 \\
Cesarean section delivery & 7 & 1 & 8 \\
$\mathbf{N}$ & 15 & 12 & $\mathbf{2 7}$ \\
\hline
\end{tabular}

*Multiple responses

Two-thirds of FWVs reported sending referral cases to the Medical College Hospital and the Upazila Health Complex. Referring clients to the District Hospital and Mother and Child Welfare Center was very uncommon in both districts. None of the FWVs in Chittagong district referred a client to qualified doctor's chamber or private clinic (Table 21). For appropriate referral, FWVs should know which facilities provide comprehensive emergency obstetric care. 
Table 21: Facilities to which clients are referred (number)

\begin{tabular}{llrrr}
\hline Referral facilities* & Chittagong & Munshiganj & Total \\
Public & Medical College Hospital & 11 & 7 & 18 \\
& District Hospital & 1 & 3 & 4 \\
& Mother and Child Welfare Center & 1 & 1 & 2 \\
& Upazila Health Complex & 8 & 9 & 17 \\
Private & Private hospital/clinic/qualified & 0 & 3 & 3 \\
N & doctor's chamber & 15 & 12 & 27 \\
\hline
\end{tabular}

*Multiple responses

\section{Problems Faced by Providers in Performing Job}

Given their long experience, FWVs were well aware of the need to strengthen the UHFWC for providing delivery services. Table 22 provides a list of obstacles that FWVs encountered in providing services at the facility. Inadequacy in human resources, lack of delivery instruments, irregular supply of electricity in labor room (or load-shedding), and bad condition of labor room were widely mentioned. Half of FWVs mentioned lack of training. Problems that hamper service delivery are more pervasive in Chittagong than in Munshiganj.

Table 22: Problems experienced by FWVs while working in the facility (number)

\begin{tabular}{lrrr}
\hline Problems* & Chittagong & Munshiganj & Total \\
Lack of staff & 11 & 5 & 16 \\
Lack of training & 8 & 5 & 13 \\
Bad condition of labor room & 7 & 8 & 15 \\
Lack of delivery instruments & 10 & 11 & 21 \\
Irregular electricity supply in labor room & 13 & 11 & 24 \\
Client flow & 5 & 0 & 5 \\
Lack of motivation for staff & 2 & 0 & 2 \\
Poor working environment & 3 & 0 & 3 \\
$\mathbf{N}$ & 15 & 12 & 27 \\
\hline
\end{tabular}

*Multiple responses 


\section{DISCUSSIONS AND RECOMMENDATIONS}

Several gaps in the existing capacity of UHFWCs for providing round-the-clock delivery services were identified through the situation analysis. Necessary inputs are broadly described in five categories: physical infrastructure, human resources, equipment and supplies, management, and referral.

\section{Physical Infrastructure}

Three types of UHFWCs operated by the DGFP were found. More than 60 percent of UHFWCs were one storied, 32 percent were two storied and only 5 percent were three storied. Largely, UHFWCs had separate rooms for the staff along with delivery and recovery rooms. Rooms are not fully furnished, nevertheless service provision is possible.

In some cases, infrastructure was in poor condition. There was lack of essential utilities, e.g., supply of water and electricity. Toilet facilities were somewhat satisfactory. On average, there were three toilets in each facility and one-fifth had separate toilets for males and females.

\section{Human Resources}

- Existing human resources are not adequate for performing delivery services at the UHFWC, nor for providing round-the-clock services. FWVs did not have adequate training to provide normal delivery services. Only half of the FWVs had training on midwifery and a few FWVs were trained on active management of the third stage of labor. Not all FWVs had adequate knowledge of obstetric danger signs. Largely, FWVs were not skilled to use partograph.

- Identification of danger signs of pregnancy and conditions for high-risk pregnancy is dependent on the correct knowledge and skills of providers. The composite skills score reveals inadequate knowledge of FWVs on pregnancy and delivery complications. The assessment also reveals an inadequacy in the skills of FWVs in the management of essential obstetric complications. Gaps in knowledge and skills for treating obstetric complications can be addressed through refresher training and technical monitoring.

- In essential counseling skills, e.g., general health counseling, birth planning counseling and advice during discharge after delivery, FWVs failed to score a high competency level. Providers need counseling training as part of the program and professional monitoring from the higher level.

- There is a serious deficiency in knowledge and skills of basic neonatal care among FWVs (a maximum composite score of 0.50 out of 1.00). Training on "essential newborn care" and professional monitoring from higher level are the possible avenues to address inadequacy in FWVs' knowledge and skills on newborn care.

- FWVs are largely non-residential despite having options for accommodation in the facility building. It is necessary to ensure that FWVs who are the first contact for pregnant women stay at the residence attached to the UHFWC in order to be able to provide 24-hour normal delivery services. 


\section{Equipment and Supplies}

- Equipment and logistics in the labor and recovery rooms were not fully available in the facilities. These shortcomings need attention as these two rooms are critical for providing normal delivery services.

- In a few UHFWCs, there is a rest room or post-operative room for IUD/MR/delivery clients. None of the UHFWCs reported having a furnished recovery room. A recovery room with at least two beds is necessary to provide round-the-clock delivery services.

- Another major problem for labor room is that only a few UHFWCs had a generator as an alternate source of electricity. For 24-hour services, it is necessary to ensure uninterrupted electricity.

- BCC materials on maternal health, such as flipchart, brochure, poster and wall writing, were found inadequate. These materials are important tools to raise awareness among service recipients.

\section{Management}

- Encouragingly, monthly reporting from UHFWCs to higher levels was found regular in all facilities.

- About 80 percent of the unions have a Union Health and Family Planning Committee (UHFPC), but only 10-18 percent of those committees were found functional where monthly meetings were held, mostly irregularly. None of the committees was empowered to oversee the activities of the UHFWC and to contribute to the UHFWC maintenance. Initiatives should be taken to reactivate the existing committees or expedite the process of forming committees for the UHFWCs having no such committee with appropriate supervisory and financial authority.

- UHFWCs should publicize their 24-hour normal delivery services, including the names of service providers, to capture the local population's attention.

\section{Referral}

- There should be specific direction on where to refer pregnant woman for complications identified at the UHFWC. UHFWCs should have a functional linkage with both basic emergency obstetric and newborn care (EmONC) and comprehensive EmONC facilities for referring complicated cases where appropriate. All Upazila Health Complexes, which are the first referral center for FWVs posted at UHFWCs, should be strengthened with appropriate human resources and equipment to conduct cesarean deliveries and provide emergency obstetric care. Currently, one-third of the Upazila Health Complexes conduct cesarean deliveries and provide EmONC. 


\section{WAY FORWARD}

Limited availability of FWVs at the UHFWC (4 out of 6 working days), who are the only providers for delivery services, is the key programmatic challenge to provide 24-hour normal delivery services from the UHFWC. Moreover, FWVs are not skilled to provide basic EmONC. The UHFWC requires a provider who should be adequately trained in midwifery care to address essential functions of EmONC and to make referral to the Upazila Health Complex and higher-level facilities for complications management and cesarean deliveries. A new cadre of "midwife" can be created to address the maternal health care needs of the growing female population in rural areas.

In rural Bangladesh, more than half of deliveries take place at home while union-level facilities remain underutilized. It has been estimated that approximately 400 child births take place in a union per year of which 15 percent require cesarean sections from higherlevel facilities. As per global standard protocol, a trained mid-level provider (e.g., FWV at the UHFWC) can perform 175 deliveries annually, which comprises half of the normal deliveries of a union. There is no alternate to increasing facility-based delivery in rural areas to reduce maternal health risks; therefore, it is necessary to strengthen UHFWCs with skilled human resources, service provision, logistics and supplies, and local level management. 